\title{
Hyperglycaemia and Diabetic Microangiopathy
}

\author{
M. D. Siperstein ${ }^{1}$, K. R. Feingold ${ }^{2}$, and P. H. Bennet ${ }^{3}$ \\ ${ }^{1}$ Department of Medicine, University of California, San Francisco, ${ }^{2}$ Metabolism Section, Veterans Administration Hospital, \\ San Francisco, California, and ${ }^{3}$ National Institute of Arthritis, Metabolism and Digestive Diseases, Phoenix, Arizona, USA
}

In their editorial, Professor Lundbaek and his colleagues bring forth a number of noteworthy issues regarding capillary basement membrane thickening and the carbohydrate abnormalities of diabetes. Many of these points have been raised previously by Williamson [1] and no doubt some of them have concerned other investigators in this field. We are therefore pleased to attempt a clarification of these issues. Professor Lundbaek's editorial raises three critical questions:

First, is capillary basement membrane thickening present in diabetic tissues at the onset of hyperglycaemia? The editorial cites the elegant studies of Osterby [2], indicating that renal glomeruli of newly diagnosed diabetics, ages 13 to 32 years, show no detectable capillary basement membrane thickening. There is no reason, however, to expect that diabetic microangiopathy appears in all tissues simultaneously and progresses at the same rate in all diabetic capillaries. We believe that most clinicians would disagree with Professor Lundbaek's statement that "the natural history of diabetic microangiopathy is the same in the kidney and in all other organs." In 1968, we introduced the electron microscopic quantification of quadriceps capillary basement membranes (QCBM) as a means by which diabetic microangiopathy could be readily and objectively evaluated. We unexpectedly observed that in muscle, in contrast to kidney, capillary basement membranes were thickened in $90 \%$ of adult diabetics at first diagnosis; furthermore, the degree of microangiopathy varied even between different muscles of the body [3]. Only two subsequent studies have measured QCBM thickening at the onset of hyperglycaemia, and both have confirmed that the majority of adult diabetics have developed this lesion by the time that hyperglycaemia is diagnosed. Specifically, Kilo et al. [4], using glutaraldehyde fixation, found that $54 \%$ of diabetics had QCBM hypertrophy at "zero duration" of diabetes; in insulin-requiring diabetics, the figure was $71 \%$. In the NIH Cooperative Study (v. i.) $88 \%$ of newly diagnosed diabetics demonstrated QCBM thickening in osmic acid-fixed muscle [5].

The consistent finding of QCBM thickening in the majority of newly diagnosed diabetics is not too surprising. Professor Lundbaek is correct that with or renal changes at first diagnosis; however, with more modern techniques Malone et al. reported that fully $66 \%$ of diabetic children manifested retinopathy within the first year of diagnosis [6], and other investigators have reported that significant proteinuria is often found at the onset of diabetes, suggesting at least the presence of functional renal disease $[7,8]$.

1935 methods clinical studies failed to reveal retinal

Second, and most important, is capillary basement membrane thickening actually present in prediabetic subjects (the offspring of two diabetic parents) prior to the appearance of hyperglycaemia? Clearly, this question bears most directly on the issue of whether the microangiopathy of diabetes is secondary to, or independent of, the metabolic abnormalities of diabetes. Our 1968 study surprisingly demonstrated that over half $(53 \%)$ of prediabetic subjects, average age 43 years, had significant QCBM hypertrophy. After modifying the QCBM method, primarily by using gluteraldehyde fixation and a non-random measurement procedure, Williamson was unable to confirm this observation [9]; he further reported that the prevalence of QCBM thickening even in overt diabetics was only $53 \%$. As a result, considerable controversy has arisen regarding both the sensitivity of the QCBM method $[1,10]$ and the validity of the results obtained with this procedure [11]. 
To resolve this controversy, the NIH in 1973 set up a Cooperative Study designed primarily to answer two major questions: first, is basement membrane thickening present in prediabetic subjects, and, second, can methodologic differences account for the differences in results reported from Williamson's and our laboratories? The NIH [5] has now reported that although the mean QCBM width was not significantly different between their prediabetic and normal subjects, with osmic acid-fixed tissues a highly significant proportion $(29 \%)$ of prediabetic subjects (average age 27 years) had QCBM hypertrophy. Moreover, since QCBM thickening in prediabetics is age dependent [5], it is noteworthy that the NIH found that by average age 38 years, $54 \%$ of the prediabetic subjects had QCBM thickening, a percentage that agrees almost exactly with our $53 \%$ figure reported a decade earlier.

The importance of methodology in assaying QCBM width is indicated by the NIH finding that glutaraldehyde fixation resulted in a far lower rate of detecting QCBM thickening in all diabetics (68\% vs. $92 \%$ ), recent-onset diabetes ( $25 \%$ vs. $88 \%$ ), and prediabetic subjects $(6 \%$ vs. $29 \%$ ) than did fixation in osmic acid. The NIH concluded that "It appears that the Siperstein method detected a higher proportion of diabetics with basement membrane thickening and also identified $29 \%$ of prediabetics as abnormal." ..."The proportion of prediabetics and diabetics identified by using osmic acid fixation was greater than with glutaraldehyde regardless of the method of measurement." The NIH study has demonstrated that differences in methodology, far from being minor, probably account for the failure of studies using gluteraldehyde-fixed tissue to observe QCBM thickening in prediabetic subjects.

The presence of QCBM hypertrophy in prediabetics, observed by this laboratory and by the NIH, again has been confirmed in the recent report of Camerini-Davalos et al. [12]. In that study, highly significant $(\mathrm{p}<0.01)$ mean QCBM thickening was observed in osmic acid-fixed tissues from 30 prediabetic subjects. Moreover, $33 \%$ of the prediabetics had diffuse and/or segmental basement membrane thickening.

In summary, the results from this laboratory [3], those of the NIH Cooperative Study [5], and most recently the data of Camerini-Davalos [12] all have indicated that $\mathrm{QBCM}$ thickening is present in older prediabetic subjects; QCBM thickening, therefore, seems unlikely to be the direct consequence of hyperglycaemia per se. It is always possible, of course, that subtle and as yet undetected metabolic disturbances related to diabetes could be present in prediabetics for some time before unequivocal evi- dence of impaired glucose tolerance. These findings clearly support the suggestion that hyperglycaemia is not prerequisite to diabetic microangiopathy.

The third question is: Does QCBM thickening progress with duration of hyperglycaemia? There is no unanimity on this point, which fortunately, however, has little bearing on the pathogenesis of diabetic microangiopathy. In muscle we observed no significant progression of QCBM thickening until the twentieth year of diabetes [3]. The data of Kilo (4), on the other hand, demonstrated some progression of basement membrane thickening in diabetics over age 40 . In their adult diabetics ${ }^{1}$, Pardo et al. [14] and Danowski et al. [15] demonstrated, respectively, an $88 \%$ and $100 \%$ prevalence of QCBM thickening and found no effect of duration upon the progression of the lesion. The NIH Cooperative Study, while observing no increase in the prevalence of QCBM thickening, did note progression of QCBM width with duration of hyperglycaemia.

But the finding of a correlation between duration of hyperglycaemia and degree of basement membrane thickening in no way supports the conclusion, implied in Professor Lundbaek's editorial, that hyperglycaemia causes basement membrane thickening. As noted above, our original report [3], that of Kilo et al. [4], the NIH study [5] and the study of Camerini-Davalos et al. [12] all have demonstrated QCBM thickening either prior to or at the time of onset of hyperglycaemia; hence a further progression of the lesion may mean equally well that the hyperglycaemia aggravates the already thickened basement membrane, that the two abnormalities are both the result of a common, primary metabolic defect, or even that basement membrane thickening causes the hyperglycaemia of diabetes. Even a strong and consistent correlation between QCBM thickening and duration of hyperglycaemia, therefore, would in reality be of little help in relating microangiopathy causally to the metabolic abnormalities of diabetes.

Two minor points raised in the editorial can be answered briefly. Professor Lundbaek notes an apparent inconsistency between the values presented in two tables of our original study [3]. As stated in that report [3] and subsequently [16], one of these tables presented a preliminary methodologic study demonstrating the close reproducibility of QCBM measurements; the other tables, representing normal, dia-

\footnotetext{
1 In analyzing QCBM data it is important to distinguish results in adults from those obtained during the first two decades of life. In contrast to adults, younger diabetics ages 11-20 have only a $50 \%$ incidence of QCBM hypertrophy, which is, however, frequently present at the time of acute onset of their disease [13]
} 
betic and prediabetic QCBMs, reflected different biopsies, different patients, or different magnification factors. Similarly, for unknown technical reasons, all the values obtained with osmic acid fixation for normal, prediabetic and diabetic subjects in the NIH study [5] were higher than those obtained earlier [3]. For this reason, any studies of QCBM width should include - as in all biological studies - control determinations carried out concomitantly.

Finally, we do not believe that the clinical implications of these QCBM results are as dire as Professor Lundbaek has implied. As emphasized in a recent editorial [17], the QCBM studies represent but one approach to the complex question of the relationship of diabetic microangiopathy to hyperglycaemia. While we believe that there is at present no convincing evidence that rigid control of blood glucose will prevent the vascular complications of diabetes, we wish to emphasize again that until this question is finally resolved, one should attempt to maintain the best possible control of glucose levels in the diabetic patient. The physician should not attempt to do so, however, at the severe price of iatrogenic hypoglycaemia, on the still unproved assumption that the microangiopathy is secondary to the carbohydrate disturbances of diabetes mellitus.

Acknowledgement. The work by Dr. Siperstein was supported by the Medical Research Service of the Veterans Administration and by United States Public Health Service Program Project grant HL06285 .

\section{References}

1. Williamson, J. R., Kilo, C.: Current status of capillary basement membrane disease in diabetes mellitus. Diabetes $\mathbf{2 6}$, 65-73 (1977)

2. Østerby Hansen, R: A quantitative estimate of the peripheral glomerular basement membrane in recent juvenile diabetes. Diabetologia 1, 97-100 (1965)

3. Siperstein, M. D., Unger, R. H., Madison, L. L.: Studies of muscle capillary basement membranes in normal subjects, diabetic, and prediabetic patients. J. Clin. Invest 47, 1973-1999 (1968)

4. Kilo, C., Vogler, N., Williamson, J. R.: Muscle capillary basement membrane changes related to aging and to diabetes mellitus. Diabetes 21, 881-905 (1972)

5. Aronoff, S. L., Bennett, P. H., Siperstein, M. D., Williamson, J. R.: Muscle capillary basement membrane thickening in prediabetic and diabetic Pima Indians. Presented at the First
International Symposium on the Biology and Chemistry of Basement Membranes, Philadelphia, Pa., Nov. 29 - Dec. 1, 1976

6. Malone, J. I., Van Cader, T. C., Edwards, W. C.: Diabetic vascular changes in children. Diabetes 26, 673-679 (1977)

7. Keen, H., Chlouverakis, C., Fuller, J., Jarrett, R. J.: The concomitants of raised blood sugar: studies in newly-detected hyperglycaemics. II. Urinary albumin excretion, blood pressure and their relation to blood sugar levels. Guy's Hosp. Rep. 118, 247-254 (1969)

8. Hemmingsen, L., Hoiby, N., Kragh-Sorensen, P.: Protein and LDH-isoenzyme pattern of the urine from patients with diabetes mellitus determined by disc-electrophoresis. Diabetologia 6, 512-518 (1970)

9. Williamson, J. R., Vogler, N. J., Kilo, C.: Basement membrane thickening in muscle capillaries. Observations on diabetics and non-diabetics with both parents diabetic. In: Endocrinology, Proc. 4th International Congress of Endocrinology, Washington, D. C., 1972. Ebling, F. J. G., Henderson, I. W. (Eds.), Scrow, R. O. International Congress Series no. 273, pp. 1122-1126. Amsterdam: Excerpta Medica 1973

10. Siperstein, M. D.: Diabetic microangiopathy. West. J. Med. 121, 404-412 (1974)

11. Williamson, J. R., Rowold, E., Hoffman, P., Kilo, C.: Influence of fixation and morphometric technics on capillary basement-membrane thickening prevalence data in diabetes. Diabetes 25, 604-613 (1976)

12. Camerini-Davalos, R.A., Oppermann, W., Rebagliati, H., Glasser, M., Bloodworth, J. M. B.: Muscle capillary basement membrane width in genetic prediabetes. Program, 60th Annual Meeting of The Endocrine Society, 1978, 167 A (abstract)

13. Raskin, P., Marks, J. F., Burns, H., Jr., Plumer, M. E., Siperstein, M. D.: Capillary basement membrane width in diabetic children. Am. J. Med. 58, 365-372 (1975)

14. Pardo, V., Perez-Stable, E., Alzamora, D. B., Cleveland, W. W.: Incidence and significance of muscle capillary basal lamina thickness in juvenile diabetes. Am. I. Pathol. 68, 67-77 (1972)

15. Danowski, T. S., Fisher, E. R., Khurana, R. C., Nolan, S., Stephan, T.: Muscle capillary basement membrane in juvenile diabetes mellitus. Metabolism 21, 1125-1132 (1972)

16. Siperstein, M. D.: The relationship of carbohydrate derangements to the microangiopathy of diabetes. In: XIII Nobel Symposion "Pathogenesis of Diabetes Mellitus", Stockholm, Sweden. Cerasi, E., Luft, R. (Eds.), p. 81. Stockholm: Almqvist \& Wilksell 1970

17. Siperstein, M. D., Foster, D. W., Knowles, H. C., Jr., Levine, R., Madison, L. L., Roth, J.: Control of blood glucose and diabetic vascular disease. N. Engl. J. Med. 296, 1060-1063 (1977)

Received: August 2, 1978

Dr. M. D. Siperstein

Department of Medicine

Veterans Administration Hospital

San Francisco, CA 94121

USA 